\title{
Refractory Lymphoplasmacytic Lymphoma
}

National Cancer Institute

\section{Source}

National Cancer Institute. Refractory Lymphoplasmacytic Lymphoma. NCI Thesaurus.

Code C8652.

Lymphoplasmacytic lymphoma resistant to treatment. 